\title{
DRIVER ALERTNESS DETECTION USING OPENCV IN PYTHON
}

\author{
Prof. Dnyaneshwar Kanade, Aseem Patil, Venugopal Bang, Maithili Jayale, Darshan Dodal, Rutik Katkamvar \\ Department of Electronics Engineering, Vishwakarma Institute of technology, \\ Pune, Maharashtra, India
}

\begin{abstract}
One of the major technical challenges in the automotive industry is the development of safety features to prevent drunk and drowsy driving. Driving while drunk or drowsy, especially in modern age, is a major reason behind road accidents. Driving when drowsy can result in a higher risk of crash than in alert condition. Therefore, by using assistive systems to monitor driver's level of alertness can be of significant help in prevention of accidents. This paper aims towards the detection of driver's drowsiness using the visual features approach along with drunk detection using alcohol sensor. Driver drowsiness is based on real-time detection of the driver's head, face and mouth. The system will also have an alcohol detection sensor which will determine whether the driver is drunk or not, thus covering the major reasons behind road accidents.
\end{abstract}

Keywords $\quad$ - $\quad$ Vehicles, Face, Feature extraction, Mouth, Visualization, Safety, Monitoring

\section{INTRODUCTION}

Drowsiness is a state resulting in reduction of consciousness caused due to lack of sleep or fatigue. Due to the drowsiness, drive loses control of the vehicle which may deviate him/her from the road and results in severe accidents. [3]According to the WHSTA organization statistics, the major factor causing accidents is sleepiness of driver. However, over the years, with the increasing growth rate of motorization accompanied by road network expansion and urbanization, India is facing serious impacts on road safety levels. In India the total number of road accidents is increased by 3 per cent from 4, 90,400 in 2014 to 5, 01,424 in 2016. The road accident data analysis of 2015 reveals that about 1374 accidents and 300 deaths take place every day on Indian roads. Few of these major causes for this huge loss are alcohol consumption while driving and drowsiness of driver. In order to minimize these huge number of accidents, advanced driver take assistance techniques can be used. For this the driver is monitored using two ways: direct and indirect. [5] Direct monitoring technique consists of head movement, facial expressions captured using sensors like camera. Driver activities and his/her response to specific situation are included in indirect techniques for monitoring drowsiness. A series of actions performed by driver while driving involves eye activities, frequency and the amount of time for which eyes were closed, head displacement with respect to the center of gravity assists in detecting the driver's current

\section{SYSTEM ARCHITECTURE}

The architecture of the system consists of various phases.

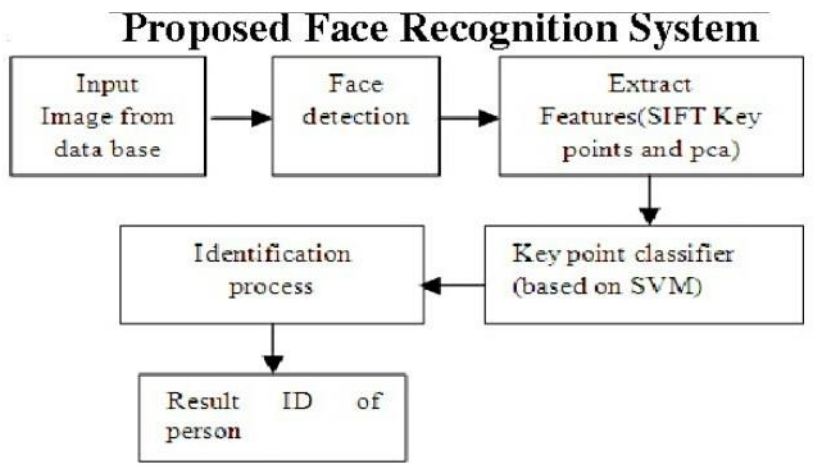

Fig 1.1 Block Diagram of facial recognition

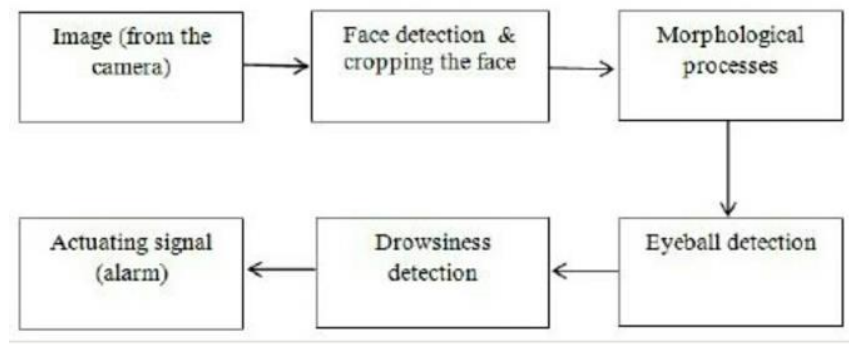

Fig 1.2 Block Diagram of the system proposed

\section{IMPLEMENTATION}

\section{A. Image Capturing:}

This is the initial stage of the system. A setup is made and optimized for current user. Successful head location of the driver is the key stage. [4] If the driver's head is successfully 


\section{International Journal of Engineering Applied Sciences and Technology, 2019 \\ Vol. 4, Issue 6, ISSN No. 2455-2143, Pages 72-76 \\ Published Online October 2019 in IJEAST (http://www.ijeast.com)}

located, it becomes easy to process the image and helps in identifying the current state of the driver.

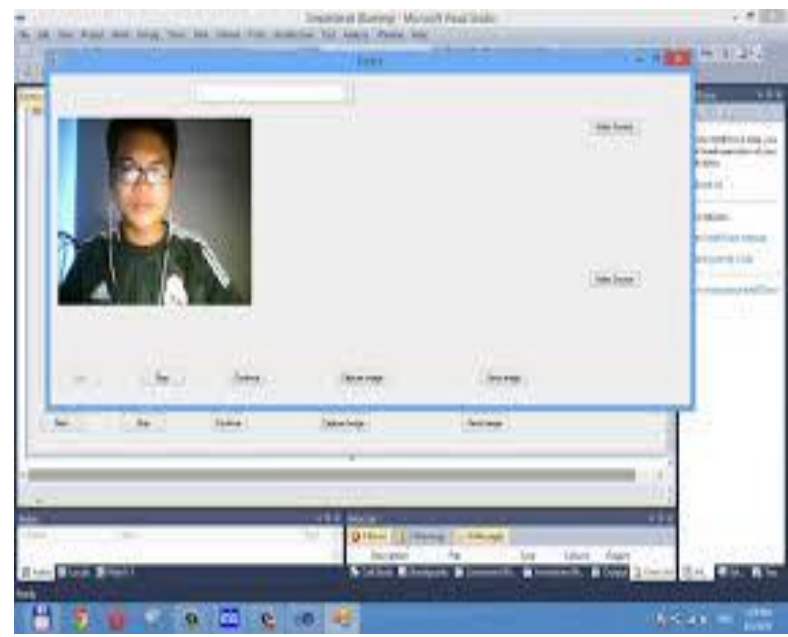

Figure 1.3 Image Capturing

The setup of our system includes: (i) extracting the driver's facial expressions and detection head movement and (ii) collecting samples of eyes open/closed. This information is further processed to analyze the state of the driver.

\section{B. Analysis:}

The system enters into analysis stage after locating the driver's head and eyes properly in image captured through camera. [7] This image is then preprocessed using various

Image Processing techniques for drowsiness detection. Various techniques involved in Image Processing are Blurring, RGB to HSV Conversion, HSV Thresholding Blob Detection. In blurring stage, the previously captured image is decomposed into pixels which spreads out and gets mixed into surrounding pixels. This obtained image consists of some unique features which can be best expressed and described using using HSV format. [1] So the RGB image format is converted using HSV format. When the pixel color range is diversed, thresholding in HSV is very useful for isolating image features that cannot be achieved by RGB thresholding. So HSV thresholding is carried out.

After image thresholding blob detection method is implemented which aims at detecting regions of image that differ in properties like brightness or color as compared to surrounding regions.

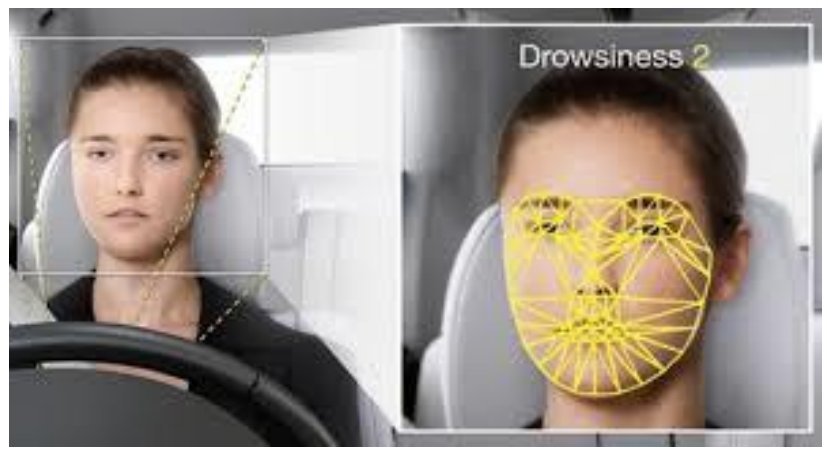

Figure 1.4 Analyzing the face by the processing system

Driver's head movement is monitored using camera which is then analyzed using Centre of Gravity to detect the driver's state. For detecting if the driver has consumed alcohol, a sensor is used which is placed right in front of the driver's face. A sensor named as MQ6 has been used in our system.

\section{Alert Stage:}

The system activates the alarm and alerts the driver if he/she is found in abnormal driving state i.e. being drowsy or if the driver has consumed alcohol. The alarm can be a buzzer, a vibrator or a previously saved audio.

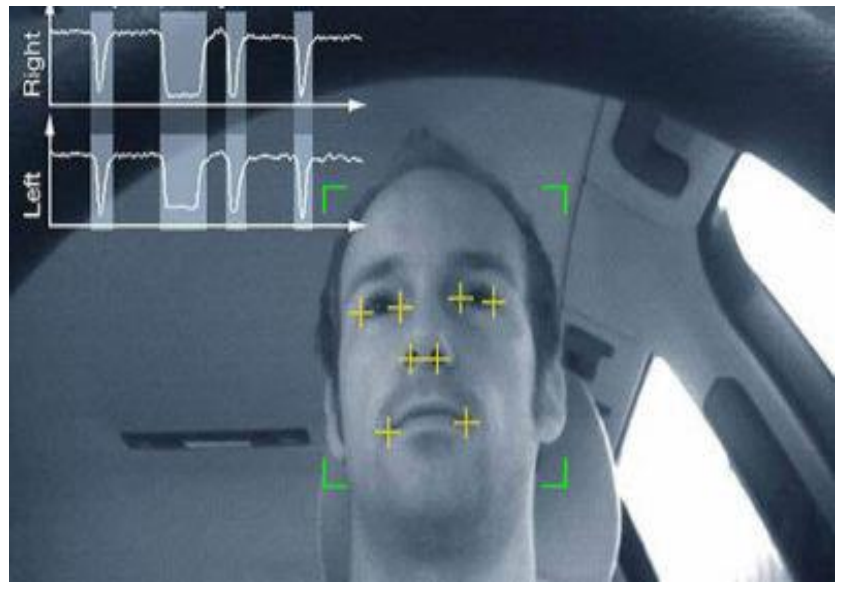

Figure 1.5 Alarming the body after detection

\section{Future Scope}

The Advanced Driver Assistance System is used to detect the drowsiness of driver. It can also be used to check if the driver is drunk or not. This system can be implemented in vehicles in real world to give provision to take live video feed of driver It can be used to send an alarm to the owner of the vehicle after detecting drowsiness Research can be done to implement the model in two-wheelers. 


\section{International Journal of Engineering Applied Sciences and Technology, 2019 Vol. 4, Issue 6, ISSN No. 2455-2143, Pages 99-102 \\ Published Online October 2019 in IJEAST (http://www.ijeast.com)}

\section{RESULT}

TABLE 1:

\begin{tabular}{|c|c|c|c|}
\hline \multirow{2}{*}{ Videos } & \multicolumn{3}{|c|}{ HaarClassifer } \\
\cline { 2 - 4 } & Total frames & Open & Ratio (\%) \\
\hline V1 & 189 & 158 & 83.598 \\
\hline V2 & 224 & 186 & 83.036 \\
\hline V3 & 182 & 150 & 82.410 \\
\hline V4 & 211 & 178 & 84.360 \\
\hline V5 & 252 & 206 & 81.746 \\
\hline V6 & 176 & 144 & 81.818 \\
\hline V7 & 192 & 157 & 81.771 \\
\hline V8 & 222 & 184 & 82.883 \\
\hline
\end{tabular}

Table 1: Table of Drowsiness State. By using the various parameters in Open $\mathrm{CV}$ we could conclude that the accuracy is nearly $94.6 \%$. The reason for such an accuracy was the fact of using real drowsy people as data samples.

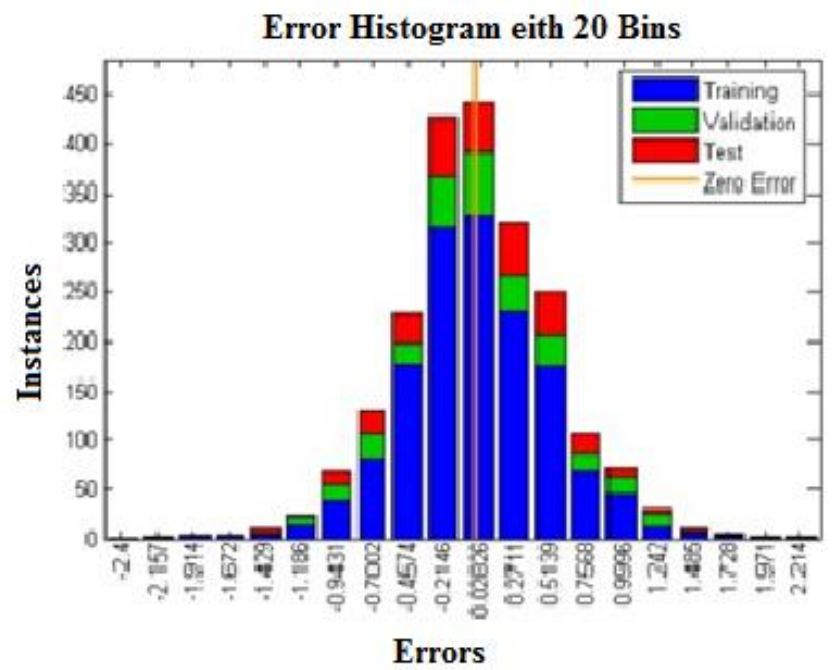

Figure 1.6 Alert versions during detection of drowsiness and there evaluation and there plotting in a bar graph.
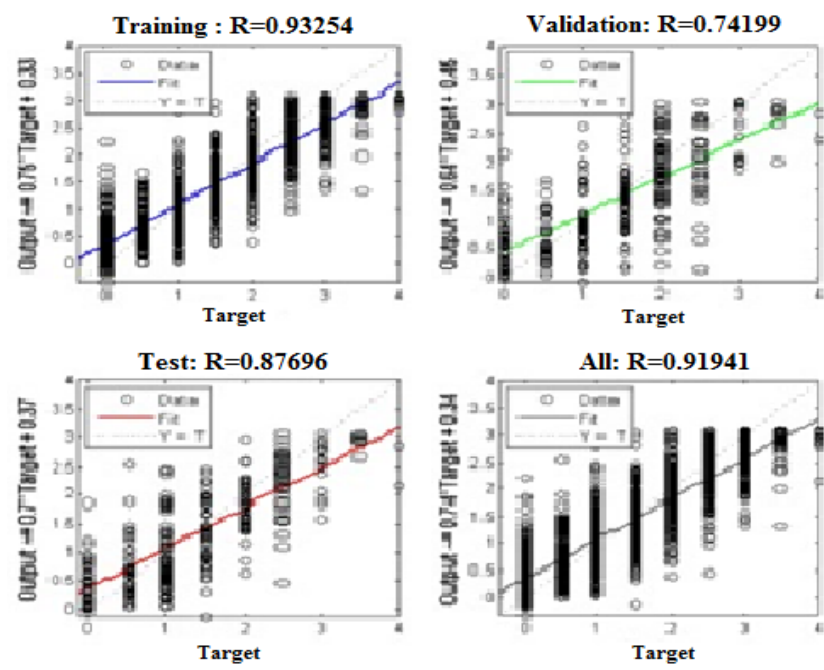

Figure 1.7 Using different datasets for implementation of the system at different circumstances and combining them and checking both of there test as well as train accuracies.

The accuracies comes as $93.25 \%$ as the training accuracy and the test accuracy comes as $87.69 \%$, along with the validation accuracy coming to be as $74.19 \%$.

\section{CONCLUSION}

This paper has proposed a system for assisting driver to avoid major accidents caused due to drowsiness of driver and alcohol consumption by assisting his/her state. State of driver is identified using algorithms related to image processing. [9]A buzzer is used to alert the driver if he/she is drowsy. With reference to the center of gravity the position of driver's head is determined and accordingly the current state of driver is identified. The movement of head is captured using a camera of appropriate resolution. A system gives extra feature of yawning detection. If a driver yawns more frequently than also an alarm is generated. A sensor is used to detect whether the driver is drunken or not. There should be proper distance between the sensor and the driver for accuracy. To inspire the driver to reach destination safely the alarm is generated which can be in the form of audio or vibration. Although there is need for more research, the proposed system can contribute effectively in detecting the driver's state and highly decrease the frequency of road accidents.

\section{REFERENCES}

[1] D. Tran, E. Tadesse and W. Sheng, Y. Sun, M. Liu and S. Zhang, "A Driver Assistance Framework Based on Driver Drowsiness Detection "The 6th Annual IEEE International Conference on Cyber Technology in 


\section{International Journal of Engineering Applied Sciences and Technology, 2019 \\ Vol. 4, Issue 6, ISSN No. 2455-2143, Pages 72-76 \\ Published Online October 2019 in IJEAST (http://www.ijeast.com)}

Automation, Control and Intelligent Systems June 19-22, 2016.

[2] Nila Novita Sari and Yo-Ping Haung "A Two-Stage Intelligent Model To Extract Features From PPG for Drowsiness Detection” 2016 International Conference on System Science and Engineering (ICSSE) National Chi Nan University July 7-9, 2016.

[3] Aleksandar, Oge Marques and Borko Furht "Design and Implementation of a Driver Drowsiness Detection System A Practical Approach".

[4] J. Ahmed, Jain-Ping Li, S. Ahmed Khan, R. Ahmed Shaikh "Eye Behavior Based Drowsiness Detection System".

[5] Anjali K U, Athiramol K Thampi, Athira Vijayaraman, Franiya Francis M, Jeffy James N, Bindhu K Rajan “ RealTime Nonintrusive Monitoring and Detection of Eye Blinking in View of Accident Prevention Due to Drowsiness" 2016 International Conference on Circuit ,Power and Computing Technologies[ICCPCT]. (2002).

[6] A. Mittal, K. Kumar, S. Dhamija, M. Kaur "Head Movement-Based Driver Drowsiness Detection: A Review of State-of-Art Techniques" 2nd IEEE International Conference on Engineering and Technology(ICETECH) March 17-18, 2016

[7] Yuichi Saito, Makoto Itoh, Toshiyuki Inagaki, "Driver Assistance System With a Dual Control Scheme: Effectiveness of Identifying Driver Drowsiness and Preventing Lane Departure Accidents"IEEE Transactions on Human-Machine Systems March 21, 2016.

[8] J. Dai, J. Teng, X. Bai, Z. Shen, and D. Xuan,"Mobile phone based drunk driving detection", in proc.IEEE health NO PERMISSION, Munich, Germany, Mar.2010,pp 1-8.

[9] K. Livescu, O. Cetin, M. Hasegawa-Johnson, S. King, C. Bartels, N. Borges, A. Kantor, P. Lal, L. Yung, A. Bezman, S. DawsonHaggerty, B. Woods, J. Frankel, M. Magami-Doss, and K. Saenko, "Articulatory featurebased methods for acoustic and audio-visual speech recognition: Summary from the 2006 jhu summer workshop," in Proc. IEEE ICASSP, vol. 4, Honolulu, HI, Apr. 2007, pp. 621-624. 